\title{
Giant bilateral sporadic renal angiomyolipoma
}

\author{
Kiyoshi Shikino MD PhD, Masatomi Ikusaka MD PhD
}

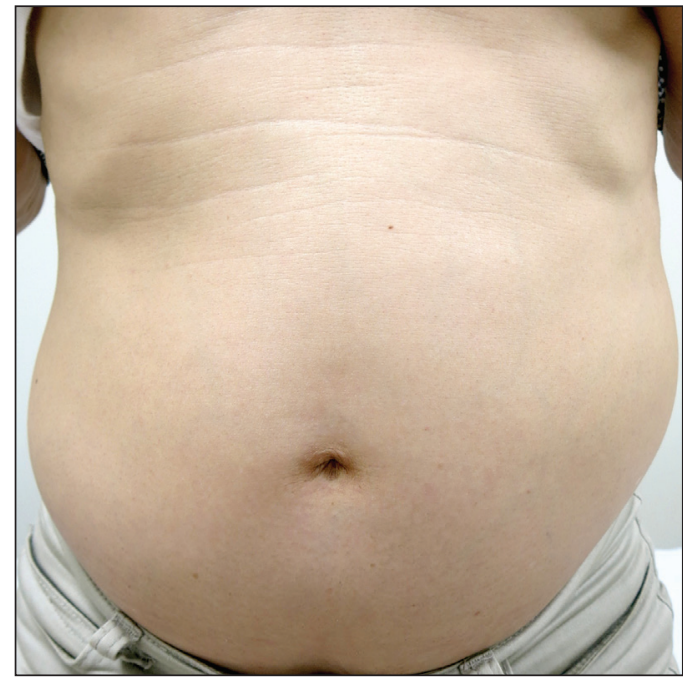

Figure 1: Distension of the abdomen in a 64-yearold man, with palpable masses in both flanks.

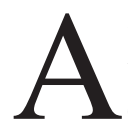

64-year-old man presented to our hospital with a 15-year history of slow, progressive distension of the abdomen. On physical examination, palpable masses in both flanks were noted (Figure 1). Results of serum creatinine and urinalysis testing were normal. Contrast-enhanced computed tomography (CT) showed large bilateral hypervascular renal masses at the periphery of both kidneys, with widespread fat attenuation consistent with bilateral renal angiomyolipoma (Figure 2). Incisional biopsy of the right mass excluded a diagnosis of malignancy. The patient underwent successful selective renal artery embolization and recovered without complications. One month after the procedure, follow-up enhanced CT confirmed reduction of the vascular component.

Angiomyolipoma is the most common benign renal neoplasm ${ }^{1}$ and accounts for $0.3 \%-3 \%$ of all diagnoses of renal neoplasm. ${ }^{2}$ It is most often detected incidentally. ${ }^{1}$ However, when tumours reach $4 \mathrm{~cm}$ in diameter, symptoms can develop (e.g., hemorrhage, palpable mass or symptoms related to mass effect). Twenty percent of cases of angiomyolipoma occur in patients with tuberous sclerosis complex; $40 \%-80 \%$ of patients with this condition have documented angiomyolipoma. ${ }^{3}$

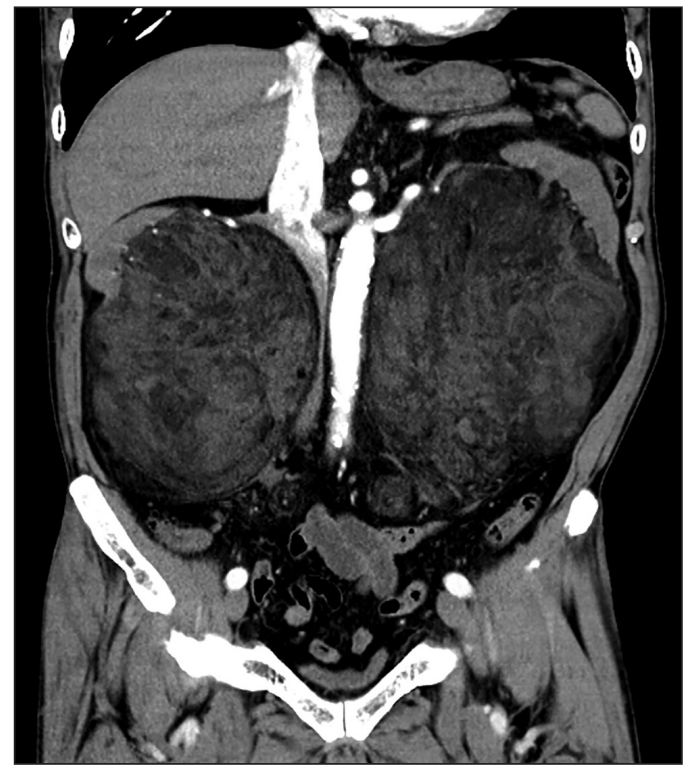

Figure 2: Coronal computed tomography image of giant bilateral renal tumours (right, $152 \mathrm{~mm} \times$ $104 \mathrm{~mm}$; left, $167 \mathrm{~mm} \times 129 \mathrm{~mm}$ ) with fat attenuation.

Our patient showed no symptoms of tuberous sclerosis complex.

Macroscopic fat within a lesion is the basis for diagnosis of angiomyolipoma (e.g., characteristic fat attenuation as seen on CT). It is important to differentiate angiomyolipoma from malignant renal disease. ${ }^{1}$ In rare instances, the smooth muscle component in angiomyolipoma may be an epithelioid variant that is fat-poor and malignant. ${ }^{3}$

Treatments preserving renal function should be considered for patients with angiomyolipoma, given its benign nature. Symptomatic lesions or tumours greater than $4 \mathrm{~cm}$ in diameter are treated with surgical resection or selective renal artery embolization. ${ }^{1}$

\section{References}

1. Halpenny D, Snow A, McNeill G, et al. The radiological diagnosis and treatment of renal angiomyolipoma-current status. Clin Radiol 2010;65:99-108.

2. Steiner MS, Goldman SM, Fishman EK, et al. The natural history of renal angiomyolipoma. J Urol 1993;150:1782-6.

3. Kattar MM, Grignon DJ, Eble JN, et al. Chromosomal analysis of renal angiomyolipoma by comparative genomic hybridization: evidence for clonal origin. Hum Pathol 1999;30:295-9.
Competing interests: None declared.

This article has been peer reviewed.

The authors have obtained patient consent.

Affiliation: Department of General Medicine, Chiba University Hospital, Chiba, Japan

Correspondence to:

Kiyoshi Shikino,

kshikino@gmail.com

CMAJ 2016. DOI: 10.1503 /cmaj.150915 